\title{
AMENDMENTS
}

\section{Author Correction: ChIP-seq of plasma cell-free nucleosomes identifies gene expression programs of the cells of origin}

Ronen Sadeh (1), Israa Sharkia, Gavriel Fialkoff(D), Ayelet Rahat, Jenia Gutin (1D, Alon Chappleboim, Mor Nitzan (1), Ilana Fox-Fisher (1), Daniel Neiman, Guy Meler (1), Zahala Kamari, Dayana Yaish, Tamar Peretz, Ayala Hubert, Jonathan E. Cohen (1), Azzam Salah, Mark Temper, Albert Grinshpun (D), Myriam Maoz, Samir Abu-Gazala, Ami Ben Ya'acov (D), Eyal Shteyer (1), Rifaat Safadi, Tommy Kaplan (1), Ruth Shemer, David Planer, Eithan Galun, Benjamin Glaser (D, Aviad Zick (1), Yuval Dor and Nir Friedman (1)

Correction to: Nature Biotechnology https://doi.org/10.1038/s41587-020-00775-6, published online 11 January 2021.

In the version of this article initially published online, the EMBL-EBI accession code now given in the first sentence of the Data Availability section was not yet available. The information has been added in the print, PDF and HTML versions of the article.

Published online: 21 January 2021

https://doi.org/10.1038/s41587-021-00831-9

(c) The Author(s), under exclusive licence to Springer Nature America, Inc. 2021

\section{Author Correction: ChIP-seq of plasma cell-free nucleosomes identifies gene expression programs of the cells of origin}

Ronen Sadeh (1), Israa Sharkia, Gavriel Fialkoff(D), Ayelet Rahat, Jenia Gutin (1), Alon Chappleboim, Mor Nitzan (1), Ilana Fox-Fisher (D), Daniel Neiman, Guy Meler (1), Zahala Kamari, Dayana Yaish, Tamar Peretz, Ayala Hubert, Jonathan E. Cohen (D), Azzam Salah, Mark Temper, Albert Grinshpun (D), Myriam Maoz, Samir Abu-Gazala, Ami Ben Ya'acov D, Eyal Shteyer (1D), Rifaat Safadi, Tommy Kaplan DD, Ruth Shemer, David Planer, Eithan Galun, Benjamin Glaser (D, Aviad Zick (1D, Yuval Dor and Nir Friedman (1D)

Correction to: Nature Biotechnology https://doi.org/10.1038/s41587-020-00775-6, published online 11 January 2021.

In the version of this article initially published online, the Venn diagram in Fig. $2 \mathrm{c}$ was generated from annotation files that had a more inclusive list of colorectal adenocarcinoma (COAD) genes, whereas elsewhere a more restrictive test was used (see Methods). The Venn diagram has been replaced with one recomputed using the more restrictive test. The error has been corrected in the print, PDF and HTML versions of the article.
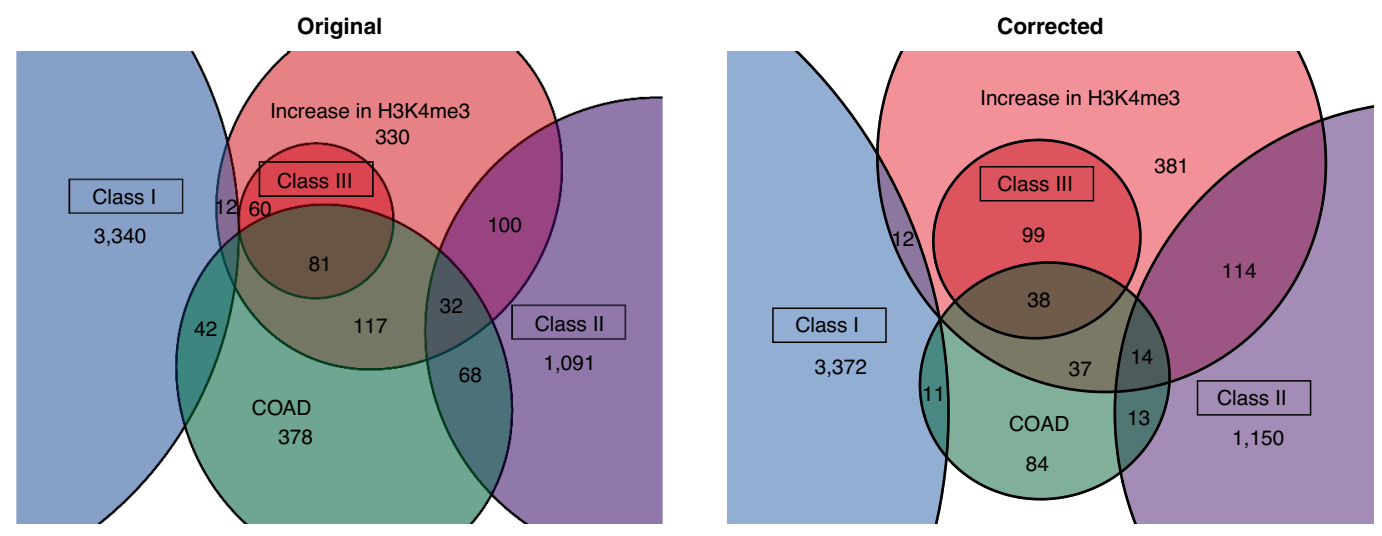

Fig. 2 | Original and corrected.

Published online: 1 March 2021

https://doi.org/10.1038/s41587-021-00853-3

(๑) The Author(s), under exclusive licence to Springer Nature America, Inc. 2021 